\title{
Skeletal complications in the course of neurofibromatosis in children
}

\author{
Elżbieta Jakubowska-Pietkiewicz, Elżbieta Woźniak, Danuta Chlebna-Sokół, Izabela Michałus
}

Department of Paediatric Propaedeutics and Bone Metabolism Diseases, Central Teaching Hospital of the Medical University of Lodz, Maria Konopnicka Paediatric Centre, Lodz, Poland

\section{ABSTRACT}

Introduction: Type 1 neurofibromatosis (NF1) belongs to the group of phacomatoses characterised by the formation of neurofibromas in the course of the nerves. In some cases, it may also lead to lesser known skeletal symptoms such as disturbances in calcium-phosphate metabolism and decreased bone mineral density. Aim of the study: The purpose of this work is to analyse possible skeletal complications in the course of neurofibromatosis, based on presentation of patients with NF1.

Material and methods: The study included 10 children diagnosed with NF1 (seven girls and three boys), aged four to 17 years. Medical examinations including evaluation of any motor system abnormalities, anthropometric measurements of body height and weight (to evaluate the respective parameters, centile charts were used from the OLAF project), and bone densitometry using dual-energy X-ray absorptiometry (DXA) were performed. Calcium and phosphate metabolisms were assessed based on laboratory tests (concentration of vitamin $\mathrm{D}-25(\mathrm{OH}) \mathrm{D}$, parathormone and alkaline phosphatase levels, and bone turnover markers).

Results: Decreased body height was found in half of the patients (5/10). Typical dysplastic bone lesions were observed in 3/10 children. On densitometry, in nine out of 10 examined children a $Z$-score decrease to $<-1.0$ was observed, and in five children this value was $\leq-2.0$. Decreased $25(\mathrm{OH}) \mathrm{D}$ concentration was found in $7 / 10$ examined children. In three out of 10 patients the osteocalcin level was increased.

Conclusions: NF1 may jeopardise normal development and function of the skeletal system in children - both of the mineral and bone metabolism. Assessment of the motor system and of the calcium and phosphate metabolism should be part of extended diagnosis and monitoring of this disease. Risk factors for bone fractures in children with NF1 are low bone mass and decreased vitamin D concentration.

\section{KEY WORDS:}

neurofibromatosis, bone markers, skeletal complications, bone mineralisation.

\section{INTRODUCTION}

Type 1 neurofibromatosis (NF1, von Recklinghausen's disease) is a disease belonging to the group of phacomatoses. It is characterised mainly by tumour formation on the course of the nerves, and in some cases it may lead to lesser known skeletal symptoms [1]. This disease is inherited as an autosomal dominant disorder, and its underlying cause is mutation and loss of function of gene

\section{ADDRESS FOR CORRESPONDENCE:}

Elżbieta Jakubowska-Pietkiewicz, Department of Paediatric Propaedeutics and Bone Metabolism Diseases, Central Teaching Hospital of the Medical University of Lodz, Maria Konopnicka Paediatric Centre, 36/50 Sporna St., 92-238 Lodz, Poland, ORCID: 0000-0002-5346-5229,

e-mail: elzbieta.jakubowska-pietkiewicz@umed.lodz.pl 
NF1 in chromosome 17 (locus 17q11.2), whose product in normal circumstances is a protein called neurofibromin, which has an inhibitory effect on cell growth [2]. The incidence of this condition is about 1 per 2500-3000 people, irrespective of ethnicity, race, and sex [3]. The description of the symptoms of the disease was first published by Friedrich Daniel von Recklinghausen in 1822 [4], and the most recent diagnostic criteria introduced in 1987 by the National Institute of Health during the NIH Consensus Development Conference are based on the occurrence of a constellation of typical clinical symptoms [5]. At present, at least two of the following symptoms are necessary for NF1 diagnosis: 1 ) $\geq 6$ café-au-lait spots with the largest diameter of $>5 \mathrm{~mm}$ in prepubertal and $>15 \mathrm{~mm}$ in postpubertal patients, 2) $\geq 2$ neurofibromas of any type or 1 plexiform neurofibroma, 3) freckles and/or discolorations in body areas not exposed to light (armpits, groins), 4) $\geq 2$ Lisch nodules (iris hamartoma tumours), 5) optic gliomas, 6) occurrence of NF1 in firstgrade relatives, and 7) presence of typical bone lesions [5].

Typical skeletal changes in the course of NF1 include low body height, distortions of spinal curvature axes in the form of scoliosis, inborn bone defects, including long bone dysplastic changes leading to recurrent non-healing fractures and false joint formation (in particular within tibiae) or sphenoid wing dysplasia [6, 7]. In recent years, occurrence of disturbances of mineral metabolism and calcium/phosphate homeostasis in these patients has also been described in the literature [8-11].

However, clinical observations suggest that the problem of bone lesions is frequently ignored, and patients without overt advanced skeletal symptoms are not always diagnosed for these problems. Therefore, we have undertaken a work whose purpose is to attract attention to the occurrence of skeletal symptoms in the course of neurofibromatosis, based on case histories and analysis of laboratory and imaging results of 10 of our patients diagnosed with NF1.

\section{MATERIAL AND METHODS}

The study included 10 children: seven girls and three boys, aged four to 17 years, who were hospitalised in the Department of Paediatric Propaedeutics and Metabolic Bone Diseases of the Medical University of Lodz for assessment of bone mineralisation and calcium/phosphate metabolism in the course of previously diagnosed NF1. After parent/legal guardian consent had been obtained, a questionnaire was completed including the history of disease symptoms and diagnostics as well as musculoskeletal symptoms, including fractures.

Medical examinations with particular emphasis on evaluation of any motor system abnormalities and anthropometric measurements of body height and weight were performed (to evaluate the respective parameters, centile charts were used from the OLAF project). Bone densitometry was also performed with use of dualenergy X-ray absorptiometry (DXA), with total body and AP spine projections (Prodigy machine, GE Lunar, USA). Densitometry results were interpreted as $Z$-score compared to age and sex reference values.

Calcium and phosphate metabolism was assessed based on laboratory tests of serum (concentration of calcium, phosphorus and magnesium, vitamin D hepatic metabolite - 25( $\mathrm{OH}) \mathrm{D}$, and parathormone and alkaline phosphatase levels) and urine (calcium/creatinine index). Bone turnover markers were also analysed, including bone formation marker - serum osteocalcin [12].

\section{RESULTS}

The clinical characteristics of studied patients, including occurrence of typical skeletal symptoms, are presented in Table 1. A detailed description of individual cases is presented below.

\section{PATIENT 1}

A four-year-old girl with NF1 meeting three criteria (approximately 20 café-au-lait spots, discolorations in armpits and groins, tibial dysplasia with formation of a false joint) diagnosed in her first year of life (Table 1). Thickened left optic nerve was shown on MRI. After the child had started to walk, forward convexity of the tibiae appeared. Then a fracture of both bones of the right lower leg occurred, with non-union of the fragments and formation of false joints in both bones, initially stabilised with intramedullary wires; however, due to a re-fracture of the right tibia and difficulties in union of bone fragments, the bone was finally stabilised with an Ilizarow device. The girl also had a history of two episodes of right fibula fracture.

\section{PATIENT 2}

A five-year-old boy with NF1 meeting three criteria (café-au-lait spots, a soft-tissue tumour of the back a neurofibroma, family history of NF1) diagnosed in his fourth year of life (Table 1). At the age of four years the patient had a head MRI scan (optic nerves no pathological lesions) and Th and L-S spine MRI scans (a tumour measuring $20 \mathrm{~mm} \times 5 \mathrm{~mm}$ was shown in the median line, at the level of the Th4 spinous processes). The tumour of the soft tissues of the back was surgically removed, and a neurofibroma was confirmed on histopathology. Due to heart rhythm disturbances the patient was diagnosed by a cardiologist as grade I/II atrioventricular block, and extrasystolia were found. The child complained of lower limb pain, especially in the evening and at night. X-rays of both were normal. 
TABLE 1. Clinical characteristics of the examined patients

\begin{tabular}{|c|c|c|c|c|c|c|c|c|c|c|}
\hline Characteristic & P1 & P2 & P3 & P4 & P5 & P6 & P7 & P8 & P9 & P10 \\
\hline $\operatorname{Sex}(M / F)$ & $\mathrm{F}$ & M & $\mathrm{F}$ & $\mathrm{F}$ & M & $\mathrm{F}$ & $\mathrm{F}$ & $\mathrm{F}$ & $\mathrm{F}$ & M \\
\hline Age (years) & 4 & 5 & 6 & 6 & 10 & 11 & 11 & 14 & 15 & 17 \\
\hline Body height in cm (centile) & $\begin{array}{c}104.9 \\
(50-75)\end{array}$ & $\begin{array}{c}117.3 \\
(75-90)\end{array}$ & $\begin{array}{c}109.7 \\
(3-10)\end{array}$ & $\begin{array}{l}104.4 \\
(<3)\end{array}$ & $\begin{array}{c}130.7 \\
(3-10)\end{array}$ & $\begin{array}{c}157 \\
(25-50)\end{array}$ & $\begin{array}{c}138.8 \\
(3-10)\end{array}$ & $\begin{array}{c}161.1 \\
(25-50)\end{array}$ & $\begin{array}{c}155.1 \\
(3-10)\end{array}$ & \begin{tabular}{|c|}
171.4 \\
$(10-25)$
\end{tabular} \\
\hline Body mass in kg (centile) & $\begin{array}{l}16.5 \\
(25)\end{array}$ & $\begin{array}{c}21.2 \\
(50-75)\end{array}$ & $\begin{array}{c}17.5 \\
(10-25)\end{array}$ & $\begin{array}{c}14 \\
(<3)\end{array}$ & $\begin{array}{c}28 \\
(10-25)\end{array}$ & $\begin{array}{c}45 \\
(25-50)\end{array}$ & $\begin{array}{c}33 \\
(25-50)\end{array}$ & $\begin{array}{c}55 \\
(50-75)\end{array}$ & $\begin{array}{c}55 \\
(25-50)\end{array}$ & $\begin{array}{c}71 \\
(50)\end{array}$ \\
\hline Age at NF1 diagnosis (years) & 1 & 4 & 2 & 2 & 6 & 2 & 4 & 12 & 15 & 16 \\
\hline BMI & $15(\mathrm{~N})$ & $15.4(\mathrm{~N})$ & $14.5(\mathrm{~N})$ & $12.8(\mathrm{~N})$ & $16.4(\downarrow N)$ & $18.3(\mathrm{~N})$ & $17.1(\mathrm{~N})$ & $21.2(\mathrm{~N})$ & $22.9(\mathrm{~N})$ & $24.2(\mathrm{~N})$ \\
\hline \multicolumn{11}{|c|}{ Clinical symptoms of NF1 } \\
\hline Café-au-lait spots & + & + & + & + & + & + & + & + & + & + \\
\hline Neurofibromas & - & + & - & - & - & + & + & - & + & + \\
\hline Freckles/discolourations & + & - & - & - & - & - & - & - & - & - \\
\hline Lisch nodules & - & - & - & - & - & + & - & - & + & + \\
\hline Optic nerve gliomas & - & - & - & + & + & - & + & - & - & - \\
\hline NF1 in relatives & - & + & + & + & - & - & + & + & + & + \\
\hline \multicolumn{11}{|c|}{ Bone lesions } \\
\hline Low height & - & - & + & + & + & - & + & - & + & - \\
\hline Scoliosis & - & - & - & - & - & + & - & - & - & - \\
\hline Long bone dysplastic lesions & + & - & - & - & - & + & - & - & + & - \\
\hline Recurrent bone fractures & + & - & - & - & - & + & - & - & + & - \\
\hline False joint & + & - & - & - & - & + & - & - & - & - \\
\hline Sphenoid wing dysplasia & - & - & - & - & - & - & - & - & - & - \\
\hline
\end{tabular}

The presence of a given symptom in the patient is marked as "+", absent symptom is marked with "-"; - male, F-female, $N$ - normal value ( \pm 2SD)

\section{PATIENT 3}

A six-year-old girl with NF1 meeting three criteria (numerous café-au-lait spots in the skin all over the body, family history of NF1, low body height) diagnosed in her second year of life (Table 1). Dysplastic lesions in the cerebellum were found on MRI scan. Convergent strabismus, and valgus knees and feet were observed in this girl; there were no bone fractures. The girl reported no symptoms related to the motor system. Lower than expected bone calcification and skeletal age delay by 1.5 years with respect to the chronological age were found on wrist X-ray.

\section{PATIENT 4}

A six-year-old girl with NF1 meeting four criteria (numerous café-au-lait spots in the skin all over the body, optic glioma, family history of NF1, low body height) diagnosed in her second year of life (Table 1). A congenital heart disease was also diagnosed, in the form of a mild pulmonary stenosis. Due to the low body height and body weight deficit, the girl is being treated by an endocrinologist and gastroenterologist. An incorrect body posture was observed; however, the girl reported no symptoms related to the motor system, and there were no bone fractures. Lower than expected skeleton calcification was reported on X-ray, and skeletal age was delayed with respect to the chronological age by 1.5 years.

\section{PATIENT 5}

A 10-year-old boy with NF1 meeting three criteria (numerous café-au-lait spots in the skin all over the body, a tumour of the optic chiasm and of the pons on MRI, low body height) diagnosed in his sixth year of life (Table 1). MRI scan was performed because of periodic gait problems with stumbling and because of NF1 suspicion raised by a neurologist. Neurosurgery was neither feasible nor indicated in this case. The child reported periodic lower limb and thoraco-lumbar spine pain. Based on X-rays of the wrist and of the spine, lower than expected bone calcification was shown. Skeleton of both shins was normal; there were no bone fractures.

\section{PATIENT 6}

An 11-year-old girl with NF1 meeting four criteria (large, jagged café-au-lait spots in the skin of the trunk and limbs, two neurofibromas - within the right shin and 
above the left buttock, Lisch nodules of the iris, skeletal lesions - advanced scoliosis, dysplasia of the left tibia with formation of a false joint and shortening of the left lower limb, disturbance of the axis of the right shin, and osteoporosis) diagnosed in her second year of life (Table 1). After the girl was born, electrolyte disturbances and hypertrophic clitoris were found - congenital adrenal hypertrophy was diagnosed initially (hydrocortisone treatment was used for two years, then adrenal hypertrophy was excluded in subsequent examinations and the hormonal treatment was terminated). Directly after the childbirth, bilateral equinovarus foot was found, with the deformity more pronounced on the left side. Since about the $12^{\text {th }}$ month of her life the skeletal symptoms worsened and the child did not start walking. A neurofibroma was found within the left shin, pressing and deforming the bone. Then a pathological fracture occurred and a false joint was formed. Café-au-lait spots were found (on the trunk) as well as two fibromatic lesions (on the right tibia and in the sacral bone area, on the left side). In the orthopaedic treatment, initially carried out in Italy and then in Poland, intramedullary fusion and left shin bone elongation with the Ilizarow method were used. Due to advanced scoliosis, an orthopaedic jacket was used, and the left lower limb was treated with an orthosis. Right ankle joint oedema was observed. The patient walked using crutches.

\section{PATIENT 7}

An 11-year-old girl with NF1 meeting five criteria (numerous café-au-lait spots, fine neurofibromas in the skin, optic glioma, positive family history - NF1 in the mother, low body height) diagnosed in her fourth year of life (Table 1). The child developed right eye vision impairment related to optic nerve atrophy due to a glioma in the region of the optic chiasm, as well as a divergent strabismus. The patient reported no symptoms related to the musculoskeletal system; there were no bone fractures. Faulty posture was found (rounded back, winged scapulae).

\section{PATIENT 8}

An 14-year-old girl with NF1 meeting two criteria (café-au-lait spots in the skin of the trunk and limbs, positive family history - NF1 in the mother and in a brother) diagnosed in her $12^{\text {th }}$ year of life (Table 1). Enlarged spleen was found on abdominal US, and central nervous system MRI revealed nodular lesions. The patient reported periodical knee joint pain, there were no bone fractures, and no abnormalities of body posture were observed.

\section{PATIENT 9}

A 15-year-old girl with NF1 meeting five criteria (numerous café-au-lait spots in the skin all over the body, subcutaneous nodules - neurofibromas, Lisch nodules of the iris, family history of NF1, low body height, and dysplastic changes with fractures of the right femur and tibia) diagnosed in her $15^{\text {th }}$ year of life (Table 1). In this patient, at the age of 15 years, primary fractures of the distal epiphysis of the right femur and of the proximal epiphysis of the right tibia occurred. These bones had dysplastic alterations, with cystic remodelling. Repeated fractures of the same bones occurred in the further course of the observation. On the femur X-ray, fracture fissure was visible in the border area between the distal part of the right femoral body and the distal right femur metaphysis, as well as very advanced osteoporosis of the peripheral skeleton. The child complained of periodic pain in the area of repeated fractures as well as in the area of the thoracic and lumbar sections of the spine. The patient adopted a forced lying position with contractures in the hip and knee joints. Atrophy of lower limb muscles was observed. Advanced osteoporosis and poor fracture healing in bones with cystic remodelling were observed.

\section{PATIENT 10}

An 17-year-old boy with NF1 meeting four criteria (café-au-lait spots in the skin of the trunk and limbs, numerous skin nodules - a neurofibroma confirmed on histopathology at the age of 16 years, Lisch nodules of the iris, and positive family history - NF1 in the mother and in a sister) diagnosed in his $16^{\text {th }}$ year of life (Table 1 ). Enlarged lymph nodes or neurofibromas 11-21 mm in diameter were found in the pelvis on abdominal ultrasound, and CNS MRI revealed focal nervous tissue defects up to $3 \mathrm{~mm}$ in diameter, located subcortically in the left temporal lobe. The patient reported no symptoms related to the musculoskeletal system, he was physically fit, with no history of fractures and normal body posture.

\section{EVALUATION OF ANTHROPOMETRIC PARAMETERS}

Short stature was found in the half of examined children $(5 / 10)$, and in four out of 10 of the children body height fell within in the $3-10 \mathrm{c}$ range. One female patient presented height deficit $<3 \mathrm{c}$, and only in this patient a body weight deficit was also found. In the other children the results of the anthropometric measurements were normal (Table 1).

\section{EVALUATION OF THE MINERAL METABOLISM}

Densitometry results are presented in Table 2. A decrease of at least one of the examined parameters was found in total in nine out of 10 examined children and was more frequently observed in the spine projection than in the total body projection ( $9 / 10$ vs. $2 / 5)$. Decreased $Z$-score, ranging from $<-1.0$ to -1.9 , was found in four 
children - in the spine projection in three of them and in the Total body projection in one child. Significantly decreased $Z$-score, corresponding to the diagnosis of low bone mass, i.e. ranging from $\leq-2.0$, was found in five children - in all five in the spine projection and at the same time in one of these children in the total body projection.

Quantitative ultrasound examination results are presented in Table 3. A decrease of at least one of the parameters was found in six out of seven examined children.

Selected biochemical parameters are presented in Table 4. Decreased concentration of the hepatic vitamin D metabolite $(25(\mathrm{OH}) \mathrm{D})$ was found in $7 / 10$ examined patients, and parathormone (PTH) concentration was normal in all examined patients. Elevated alkaline phosphatase activity was shown also in 7/10 patients, and in the majority of cases the increase of the activity of this enzyme accompanied the vitamin D deficit. Increased osteocalcin concentration was found in three patients.

\section{DISCUSSION}

Clinical symptoms of NF1 are spectacular, and when they appear it is not difficult to establish the diagnosis, in particular if there is a family history of the disease. However, bone lesions do not belong to the main diagnostic criteria, and if they do not cause significant motor dysfunctions, they may remain undiagnosed and untreated for a long time, which worsens further prognosis.

One of the easiest skeletal symptoms to detect and monitor is short stature. According to the literature, it is found in $14-19 \%$ of NF1 patients [13, 14]. Among our patients it was diagnosed in as many as half of the examined children $(5 / 10)$. It should be noted that the majority of these patients were in their early childhood and that
TABLE 2. The results of bone densitometry in total body and AP spine projections

\begin{tabular}{|l|c|c|c|c|}
\hline \multirow{2}{*}{$\begin{array}{l}\text { Patient no. } \\
\text { (age) }\end{array}$} & \multicolumn{2}{|c|}{ Total body } & \multicolumn{2}{c|}{ AP spine } \\
\cline { 2 - 5 } & $\begin{array}{c}\text { BMD } \\
\left(\mathrm{g} / \mathrm{cm}^{2}\right)\end{array}$ & $Z$-score & $\begin{array}{c}\text { BMD } \\
\left(\mathrm{g} / \mathrm{cm}^{2}\right)\end{array}$ & $Z$-score \\
\hline P1 (4 years) & - & - & - & $-2.1 \downarrow \downarrow$ \\
\hline P2 (5 years) & - & - & 0.663 & +0.5 \\
\hline P3 (6 years) & 0.564 & $-1.6 \downarrow$ & 0.504 & $-2.2 \downarrow \downarrow$ \\
\hline P4 (6 years) & - & - & 0.513 & $-2.1 \downarrow \downarrow$ \\
\hline P5 (10 years) & 0.713 & -0.6 & 0.573 & $-1.9 \downarrow$ \\
\hline P6 (11 years) & - & - & 0.633 & $-2.4 \downarrow \downarrow$ \\
\hline P7 (11 years) & 0.736 & -0.9 & 0.690 & $-1.6 \downarrow$ \\
\hline P8 (14 years) & 0.889 & -0.7 & 0.957 & $-1.1 \downarrow$ \\
\hline P9 (15 years) & 0.912 & $-2.1 \downarrow \downarrow$ & 0.715 & $-4.1 \downarrow \downarrow$ \\
\hline P10 (17 years) & - & - & 1.038 & $-1.5 \downarrow$ \\
\hline
\end{tabular}

Decreased values, ranging from $<1.0$ to -1.9 , are marked with an arrow pointing downwards: $\downarrow$, decreased values $\leq-2.0$ are marked with two arrows pointing downwards: $\downarrow \downarrow$

the incidence of this symptom may change after the adolescence period.

Another skeletal symptom, scoliosis, may affect 10$26 \%$ of the NF1 patients, and for this reason it is indicated that children with this diagnosis were under constant specialist observation, as is the case for our patients. Its most severe form is dystrophic scoliosis, but it was not found among our patients. It is estimated to occur in less than $10 \%$ of patients with NF1 and scoliosis [9]. It is characterised by early onset and rapid progression, it very frequently requires surgical immobilisation of the vertebrae, and, if not properly treated, it may contribute to compression fractures of the vertebrae, as well as spinal cord compression and its neurological consequences in

TABLE 3. Results of quantitative ultrasound examinations of radial and tibial bones

\begin{tabular}{|c|c|c|c|c|c|c|c|c|}
\hline \multirow{3}{*}{$\begin{array}{l}\text { Patient no. } \\
\text { (age) }\end{array}$} & \multicolumn{4}{|c|}{ Radial bone } & \multicolumn{4}{|c|}{ Tibial bone } \\
\hline & \multicolumn{2}{|c|}{ Right } & \multicolumn{2}{|c|}{ Left } & \multicolumn{2}{|c|}{ Right } & \multicolumn{2}{|c|}{ Left } \\
\hline & $\begin{array}{l}\text { SOS } \\
(\mathrm{m} / \mathrm{s})\end{array}$ & Z-score & $\begin{array}{c}\text { SOS } \\
(\mathrm{m} / \mathrm{s})\end{array}$ & Z-score & $\begin{array}{l}\text { SOS } \\
(\mathrm{m} / \mathrm{s})\end{array}$ & Z-score & $\begin{array}{l}\text { SOS } \\
(\mathrm{m} / \mathrm{s})\end{array}$ & Z-score \\
\hline P1 (4 years) & 3436 & $-2.4 \downarrow$ & 3440 & $-2.4 \downarrow$ & - & - & 3586 & -0.1 \\
\hline P2 (5 years) & 3724 & +0.2 & 3841 & +1.4 & 3617 & -0.1 & 3405 & $-2.6 \downarrow$ \\
\hline P3 (6 years) & 3453 & $-2.8 \downarrow$ & 3553 & -1.8 & 3437 & $-2.1 \downarrow$ & 3437 & $-2.1 \downarrow$ \\
\hline P4 (6 years) & 3350 & $-3.9 \downarrow$ & 3495 & $-2.4 \downarrow$ & 3379 & $-2.8 \downarrow$ & 3481 & -1.7 \\
\hline P5 (10 years) & 3802 & +0.3 & 3493 & $-2.7 \downarrow$ & 3682 & +0.1 & 3781 & +1.3 \\
\hline P6 (11 years) & - & - & - & - & - & - & - & - \\
\hline P7 (11 years) & 3643 & -1.5 & 3589 & $-2.0 \downarrow$ & 3646 & -0.4 & 3691 & +0.1 \\
\hline P8 (14 years) & 3861 & -0.2 & 3810 & -0.7 & 3597 & $-2.1 \downarrow$ & 3741 & -0.6 \\
\hline P9 (15 years) & - & - & - & - & - & - & - & - \\
\hline P10 (17 years) & - & - & - & - & - & - & - & - \\
\hline
\end{tabular}

Decreased values are marked with an arrow pointing downwards: $\downarrow$, SOS - speed of sound 
TABLE 4. Selected indicators of calcium and phosphate metabolism and bone turnover markers

\begin{tabular}{|l|c|c|c|c|}
\hline $\begin{array}{l}\text { Patient no. } \\
\text { (age) }\end{array}$ & $\begin{array}{c}25(\mathrm{OH}) \mathrm{D} \\
(\mathrm{ng} / \mathrm{ml})\end{array}$ & $\begin{array}{c}\text { PTH } \\
(\mathrm{pg} / \mathrm{ml})\end{array}$ & $\begin{array}{c}\text { Alkaline } \\
\text { phosphatase } \\
(\mathrm{IU} / \mathrm{l})\end{array}$ & $\begin{array}{c}\text { Osteocalcin } \\
(\mathrm{ng} / \mathrm{ml})\end{array}$ \\
\hline P1 (4 years) & 55.3 & 17.7 & $220 \uparrow$ & 70.7 \\
\hline P2 (5 years) & $22.9 \downarrow$ & 24.0 & $275 \uparrow$ & 58.5 \\
\hline P3 (6 years) & 36.2 & 20.4 & $320 \uparrow$ & $121.8 \uparrow$ \\
\hline P4 (6 years) & $24.4 \downarrow$ & 29.2 & $214 \uparrow$ & 10.2 \\
\hline P5 (10 years) & 39.9 & 21.3 & $159 \uparrow$ & 83.8 \\
\hline P6 (11 years) & $4.0 \downarrow$ & 35.6 & 117 & 59 \\
\hline P7 (11 years) & $19.2 \downarrow$ & 30.2 & $434 \uparrow$ & $183.2 \uparrow$ \\
\hline P8 (14 years) & $5.7 \downarrow$ & 26.6 & $224 \uparrow$ & $205.1 \uparrow$ \\
\hline P9 (15 years) & $27.8 \downarrow$ & 36.8 & 78 & 52.7 \\
\hline P10 (17 years) & $16.5 \downarrow$ & 21.7 & 119 & 55.1 \\
\hline
\end{tabular}

$25(\mathrm{OH}) \mathrm{D}$ - recommended concentration $>30 \mathrm{ng} / \mathrm{ml}$, $\mathrm{PTH}$ - reference values $15-65 \mathrm{pg} / \mathrm{ml}$, alkaline phosphatase, osteocalcin - varying reference values, depending on age and sex, decreased values are marked with an arrow pointing downwards: $\downarrow$, elevated values are marked with an arrow pointing upwards: $\uparrow$

the form of palsies $[15,16]$. Among our patients, scoliosis was present only in one girl, who needed to wear an orthopaedic jacket; however, two other patients experienced pain of the thoracic and lumbar segments of the spine.

Another skeletal disturbance is congenital tibial dysplasia, diagnosed in infancy, that initially manifests as anterolateral shin curvature. There is controversy about the pathogenesis of this deformity. The majority of authors think that the main cause of this symptom is decreased thickness of the cortical layer in the course of dysplastic remodelling of the tibia, which predisposes it to bending under the patient's own body weight, and in the further stage it may lead to pathological fractures of this bone. Repeated fractures and difficulties with their healing lead to formations of a false joint $[1,17,18]$. However, Stevenson et al., based on their own retrospective studies of more than 50 patients and PQCT (peripheral quantitative computed tomography) analysis, have come to other conclusions. According to these investigators, the anterolateral curvature of the tibiae is the result of thickening of the cortical layer of the bone and concomitant shrinkage of the marrow cavity $[7,17]$. A different opinion about the causes of these disturbances is presented by Birke et al., who suspect the presence of pseudoarthrosis, narrowings, and development of cysts in the tibia in association with a local loss of the second allele of the NF1 gene in the bone tissue in this area [18]. Orthopaedic treatment of the resulting false joints is very difficult, bone fragments frequently fail to unite, and stabilisation with an Ilizarow device is necessary [19]. This type of serious complication occurred in two of our female patients (P1 and P6).

Another bone deformity in NF1 is sphenoid wing dysplasia, occurring typically unilaterally in the area of the orbital lamina of the ethmoid or frontal bone. The lesion described above is most frequently occult and is detected during follow-up imaging studies. However, some patients present with pulsating exophthalmos or with orbital cerebral hernias [20]. This type of disturbance was not found in any of our patients. One patient (P4) presented left sided exophthalmos; however, this was associated with the presence of optic glioma and not with skull bone lesions.

The bone lesion described above, typical for NF1 patients, may also be associated with local and systemic decrease of bone mineral density assessed with densitometry. Low bone mass predisposes to osteoporosis and fractures, and in our study three patients had recurrent fractures. Among the authors of publications describing skeletal disturbances in the course of NF1 the dominant opinion is that both paediatric and adult patients with this condition have significantly decreased bone mineral density, as compared to the general population who are deemed healthy $[8,9,11]$. In our study, a decrease of BMD $Z$-score $<-2.0$ was found in half $(5 / 10)$ of the patients, including the three patients with fractures. Values ranging from $<1.0$ to -1.9 were observed in the next $4 / 10$ patients, and in total bone mineral density abnormalities were observed in nine out of 10 examined patients. We observed the lowest values in the children with fractures, including false joints, which is accordant with observations of Stevenson et al., who proved that children with NF1 with skeletal disturbances have markedly lower BMD than children with NF1 without skeletal abnormalities, and this is statistically significant [21]. The literature points also to the importance of DXA projection in patients with NF1. In this study, decreased bone mineral density was more frequent in the spine projection than in the total body projection ( $9 / 10$ vs. $2 / 5)$. This corresponds to the reports of other authors who have shown that the decrease of bone mineral density is larger in the spine projection than in the total body projection and much better correlates with bone lesions and frequently even precedes clinical manifestation in the form of fractures and deformities [22].

Another important problem in NF1 patients is inadequate vitamin D supply, measured with the concentration of the hepatic metabolite of this vitamin. In 7/10 of the patients described, $25(\mathrm{OH}) \mathrm{D}$ concentration values $<30 \mathrm{ng} / \mathrm{ml}$ were found, including four patients with a deficiency $<20 \mathrm{ng} / \mathrm{ml}$. This is probably a result of a low exposure to the sun's radiation and a diet poor in vitamin D-containing products, thus it is very important to pay attention to the treatment of vitamin D deficiency in children with known diagnosis of NF1. An additional and important argument in this issue is the discovery made by scientists from the University of Hamburg, who pointed to an inversely proportional relationship between low serum $25(\mathrm{OH}) \mathrm{D}$ concentration and presence of neurofibromas in the skin [23]. Some authors have also shown that vitamin D supplementation for a period of 1-2 years improves bone mineral density; however, these studies are based on a group of adults [24]. Thus, the above reports 
show how important serum vitamin D concentration monitoring is in patients with NF1. They also suggest its new therapeutic indications and a necessity to develop new guidelines of vitamin D supplementation in this group of patients.

A relatively poorly understood issue in the diagnostics of skeletal disturbances in children with NF1 is the assessment of bone tissue activity through measurements of bone turnover markers. According to observations of Petramal et al., patients with NF1 and skeletal disturbances present significantly lower osteocalcin concentration, as compared to those without skeletal symptoms. They suggest that osteocalcin, which is a marker of bone formation, may be a good predictive factor of development of skeletal system changes in this group of patients [25]. In our study, we did not observe decreased osteocalcin concentration in particular patients. In contrast, it was elevated in three children, but not in the patients with advanced skeletal symptoms. Nevertheless, it seems that this is a challenge deserving further efforts as new treatment options appear that may significantly modify bone turnover, which may bring hope for better quality of life of patients with severe skeletal symptoms.

\section{CONCLUSIONS}

NF1, a disease with many clinical presentations, may jeopardise normal development and function of the skeletal system in children - both of the mineral and bone metabolism. Risk factors for bone fractures in children with NF1 are low bone mass and decreased vitamin D concentration. Assessment of the motor system and of the calcium and phosphate metabolism should be part of the extended diagnosis and monitoring of this disease.

\section{ACKNOWLEDGEMENTS}

The work is financed within statutory activity no. 503/1-090-02/503-11-001.

\section{DISCLOSURE}

The authors declare no conflict of interest.

\section{REFERENCES}

1. Williams VC, Lucas J, Babcock MA, et al. Neurofibromatosis type 1 revised. Pediatrics 2009; 123: 124-133.

2. Wallace MR, Marchuk DA, Andersen LB, et al. Type 1 neurofibromatosis gene: identification of a large transcript disrupted in three NF1 patients. Science 1990; 249: 181-186.

3. Huson SM, Harper PS, Compston DA. Von Recklinghausen neurofibromatosis. A clinical and population study in south-east Wales. Brain 1988; 111: 1355-1381.

4. Reynolds RM, Browning GG, Nawroz I, Campbell IW. Von Recklinghausen's neurofibromatosis: neurofibromatosis type 1. Lancet 2003; 361: 1552-1554.
5. National Institutes of Health Consensus Development Conference Statement: neurofibromatosis. Bethesda, Md., USA, July 13-15, 1987. Neurofibromatosis 1988, 1: 172-178.

6. Alwan S, Armstrong L, Joe H, et al. Associations of osseous abnormalities in Neurofibromatosis 1. Am J Med Genet A 2007; 143A: 1326-1333.

7. Stevenson DA, Carey JC, Viskochil DH, et.al. Analysis of radiographic characteristics of anterolateral bowing of the leg before fracture in neurofibromatosis type 1. J Pediatr Orthop 2009; 29: 385-392.

8. Brunetti-Pierri N, Doty SB, Hicks J, et al. Generalized Metabolic Bone Disease in Neurofibromatosis Type I. Mol Genet Metab 2008; 94: 105-111.

9. Lammert M, Kappler M, Mautner VF, et al. Decreased bone mineral density in patients with neurofibromatosis 1. Osteoporos Int 2005; 16: 1161-1166.

10. Stevenson DA, Viskochil DH, Carey JC, et al. Pediatric 25-hydroxyvitamin D concentrations in neurofibromatosis type 1. J Pediatr Endocrinol Metab 2011; 24: 169-174.

11. Dulai S, Briody J, Schindeler A, et al. Decreased bone mineral density in neurofibromatosis type 1: results from a pediatric cohort. J Pediatr Orthop 2007; 27: 472-475.

12. Gajewska J, Ambroszkiewicz J, Laskowska-Klita T. Biochemical markers of bone turnover and their use. Med Wieku Rozwoj 1999; 3: 421-432.

13. Friedman JM. Neurofibromatosis 1: Clinical manifestations and diagnostic criteria. J Child Neurol 2002; 17: 548-554, discussion 571-572, 646-651.

14. Janus D, Wojcik M, Kalicka-Kasperczyk A, et al. Neurofibromatosis type I-the pediatric endocrinologist's point of view. Przegl Lek 2010; 67: 1155-1159.

15. Greggi T, Martikos K. Surgical treatment of early onset scoliosis in neurofibromatosis. Stud Health Technol Inform 2012; 176: 330-333.

16. Crawford AH, Herrera-Soto J. Scoliosis associated with neurofibromatosis. Orthop Clin North Am 2007; 38: 553-562, vii.

17. Stevenson DA, Zhou H, Ashrafi S, et al. Double inactivation of NF1 in tibia pseudarthrosis. Am J Hum Genet 2006; 79: 143-148.

18. Birke O, Schindeler A, Ramachandran M, et al. Preliminary experience with the combined use of recombinant bone morphogenetic protein and bisphosphonates in the treatment of congenital pseudarthrosis of the tibia. J Child Orthop 2010; 4: 507-517.

19. Trigui M, Ayadi K, Sakka M, et al. Orthopaedic manifestations of Von Recklinghausen's neurofibromatosis. Presse Med 2011; 40: e152-162.

20. Alwan S, Tredwell SJ, Friedman JM. Is osseous dysplasia a primary feature of neurofibromatosis 1 (NF1)? Clin Genet 2005; 67: 378-390.

21. Stevenson DA, Moyer-Mileur LJ, Murray M, et al. Bone mineral density in children and adolescents with neurofibromatosis type 1 . J Pediatr 2007; 150: 83-88.

22. Yilmaz K, Ozmen M, Bora Goksan S, Eskiyurt N. Bone mineral density in children with neurofibromatosis 1. Acta Paediatr 2007; 96: 1220-1222.

23. Lammert M, Friedman JM, Roth HJ, et al. Vitamin D deficiency associated with number of neurofibroma in neurofibromatosis 1 . J Med Genet 2006; 43: 810-813.

24. Schnabel C, Jett K, Friedman JM, et al. Effect of vitamin D3 treatment on bone density in neurofibromatosis 1 patients: a retrospective clinical study. Joint Bone Spine 2013; 80: 315-319.

25. Petramala L, Giustini S, Zinnamosca L, et al. Bone mineral metabolism in patients with neurofibromatosis type 1 (von Recklingausen disease). Arch Dermatol Res 2012; 304: 325-331. 